Bo Zhang - Hoi Dick Ng · John H.S. Lee

\title{
Measurement and Scaling Analysis of Critical Energy for Direct Initiation of Gaseous Detonations
}

Submitted: 6 May 2011

\begin{abstract}
In this paper, the critical energies required for direct initiation of spherical detonations in four gaseous fuels $\left(\mathrm{C}_{2} \mathrm{H}_{2}, \mathrm{C}_{2} \mathrm{H}_{4}, \mathrm{C}_{3} \mathrm{H}_{8}\right.$ and $\left.\mathrm{H}_{2}\right)$ - oxygen mixtures at different initial pressures, equivalent ratios and with different amount of argon dilution are reported. Using these data, a scaling analysis is performed based on two main parameters of the problem: the explosion length $R_{o}$ that characterizes the blast wave and a characteristic chemical length that characterizes the detonation. For all the undiluted mixtures considered in this study, it is found that the relationship is closely given by $R_{o} \approx 26 \lambda$, where $\lambda$ is the characteristic detonation cell size of the explosive mixture. While for $\mathrm{C}_{2} \mathrm{H}_{2}-2.5 \mathrm{O}_{2}$ mixtures highly diluted with argon, in which cellular instabilities are shown to play a minor role on the detonation propagation, the proportionality factor increases to $37.3,47$ and 54.8 for $50 \%, 65 \%$ and $70 \%$ argon dilution, respectively. Using the ZND induction length $\Delta_{I}$ as the characteristic chemical length scale for argon diluted or 'stable' mixtures, the explosion length is also found to scale adequately with $R_{o} \approx 2320 \Delta_{I}$.
\end{abstract}

Keywords Detonation · Critical energy · Direct initiation $\cdot$ Explosion length

PACS 47.40. Rs

\section{B. Zhang}

State Key Laboratory of Explosion Science and Technology, Beijing Institute of Technology

Beijing, 100081, China

J.H.S. Lee

Department of Mechanical Engineering, McGill University Montréal, Québec, H3A 2K6, Canada

H.D. $\mathrm{Ng}$

Mechanical and Industrial Engineering, Concordia University Montréal, Québec, H3G 1M8, Canada

Tel.: (514) 848-2424 x 3177

Fax: (514) 848-3175

E-mail: hoing@encs.concordia.ca

\section{Introduction}

The critical energy required for the direct blast initiation of a detonation in a given explosive mixture has long been considered as perhaps the most direct means of determining an explosive sensitivity [1,2]. Direct initiation of detonation, in contrast to the transition from deflagration to detonation (DDT), refers to the 'instantaneous' formation of a detonation in the asymptotic decay of the strong blast wave from a powerful ignition source [3-5]. The energy of the ignition source is thus the sole parameter which characterizes the decay of the strong blast and the detonation initiation. From blast wave theory, the characteristic length scale for the spherical blast wave is given by the explosion length, $R_{o}=\left(E_{\text {spherical }} / p_{o}\right)^{1 / 3}$. Dimensional considerations thus suggest that the explosion length must correlate with a chemical reaction length that describes the detonation structure for the direct initiation problem [6-8].

The objective of this paper is twofold: new measurement data of the critical energy for direct initiation of spherical detonations in four gaseous fuels $\left(\mathrm{C}_{2} \mathrm{H}_{2}, \mathrm{C}_{2} \mathrm{H}_{4}\right.$, $\mathrm{C}_{3} \mathrm{H}_{8}$ and $\mathrm{H}_{2}$ ) - oxygen mixtures at different initial pressures, equivalent ratios and with different amount of argon dilution are first reported. Experiments were carried out in a high pressure spherical chamber and direct initiation was achieved via a high voltage capacitor discharge. The effective spark energy was estimated from the analysis of the current output of the ignition system during the discharge. Detailed information about the experimental set-up and measurement procedure can be found in our previous publications [9-13]. Using these data, a scaling analysis is performed to investigate the relationship between the explosion length $R_{o}$ and the chemical length scale of the detonation structure. The results of the analysis appear to further contrast the direct initiation of detonations in undiluted unstable mixtures, in which cellular instability plays a prominent role and that the cell size is used to characterize the detonation structure; and in highly argon diluted mixtures where the detonation 


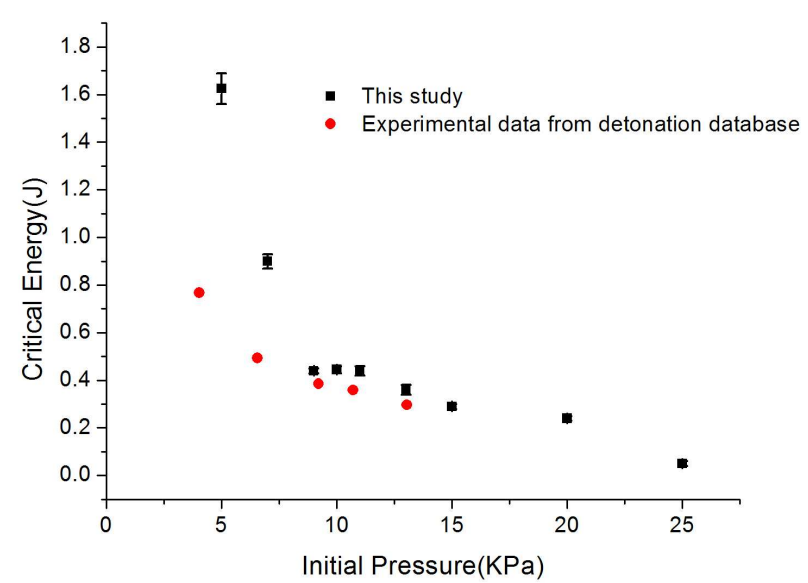

(a)

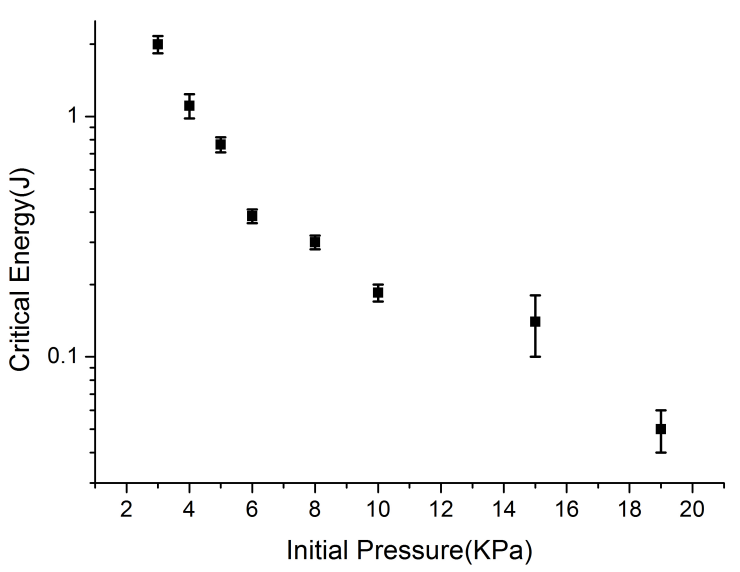

(b)

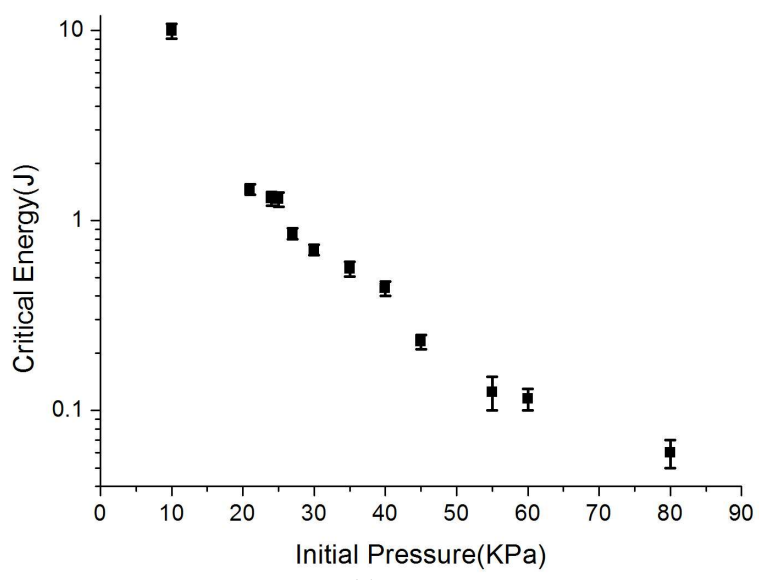

(c)

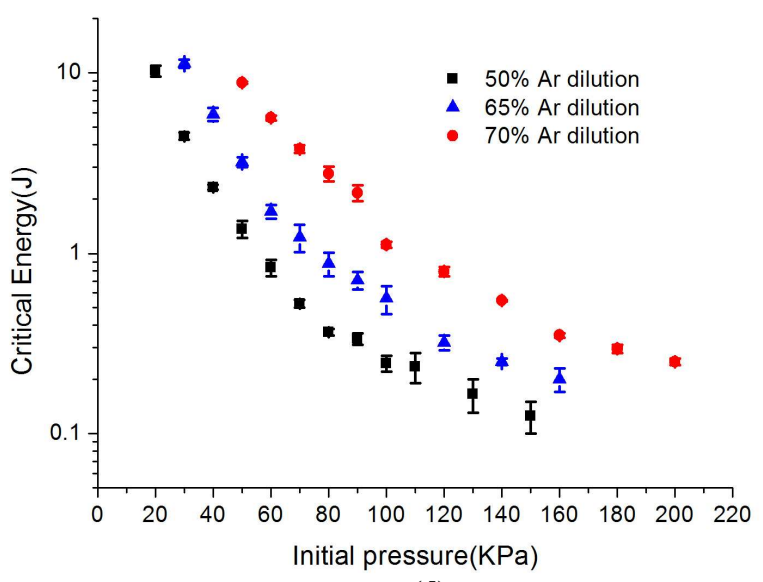

(d)

Fig. 1 Critical energy as a function of initial pressure for a) $\mathrm{C}_{2} \mathrm{H}_{2}-2.5 \mathrm{O}_{2}$; b) $\mathrm{C}_{2} \mathrm{H}_{2}-\mathrm{O}_{2}$; c) $\mathrm{C}_{2} \mathrm{H}_{2}-4 \mathrm{O}_{2}$; d) $\mathrm{C}_{2} \mathrm{H}_{2}-2.5 \mathrm{O}_{2}-50 \%$ Ar, $-65 \%$ Ar and $-70 \%$ Ar mixtures.

is relatively stable in that the reaction zone is at least piecewise laminar described by the ZND model and that the detonation structure can be characterized well with the steady ZND reaction length readily computed using the chemical kinetic data of the reaction [14-16].

\section{Results and discussion}

Figs. 1-3 summarized all the measurement results obtained in this study. The critical initiation energies of undiluted and diluted hydrocarbon-oxygen mixtures are plotted for different initial pressures and equivalent ratios. The upper and lower limits of the error bar represent respectively the last energy values used in an experiment with the same conditions at which successful and failure of direct initiation are observed. Also shown are available data from the Caltech Detonation Database [17] for comparison. In general, the present data follows and extends well those found in the Detonation Database, except there may appear a notable discrepancy for $\mathrm{C}_{2} \mathrm{H}_{4}$ results shown in Fig. 2b. Nonetheless, it should be pointed out that the initiation energy reported in [1] was not 'di- rectly' measured. The method is by first obtaining the critical tube diameter, which is then put into a semiempirical work done model to approximate the initiation energy [1]. Fig. 4 also provides new experimental data for $2 \mathrm{H}_{2}-\mathrm{O}_{2}$ mixtures at higher initial pressure than those previously published in [17] and data not reported in our previous publication [10].

Using the present critical energy data, it is possible to carry out a quantitative scaling analysis for the direct blast initiation problem. Since the strong spherical blast length scale is given by the explosion length, $R_{o}=\left(E_{\text {spherical }} / p_{o}\right)^{1 / 3}$ and the sensitivity of a detonating mixture can be described by a characteristic chemical reaction length, or equivalently the cell size $\lambda$, dimensional considerations led Lee and co-workers $[6-8]$ to propose the concept of critical explosion length scaling, whereby there should exist a proportionality law between the explosion length at critical initiation conditions, $R_{o}^{*}$ and the characteristic length scale of the self-propagating detonation. Fig. 5 illustrates this simple relationship by plotting the explosion length $R_{o}$ versus the detonation 


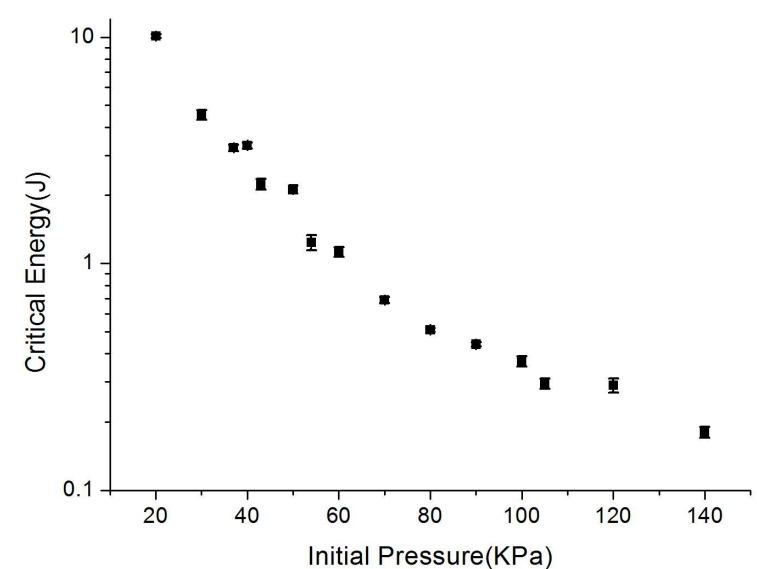

(a)

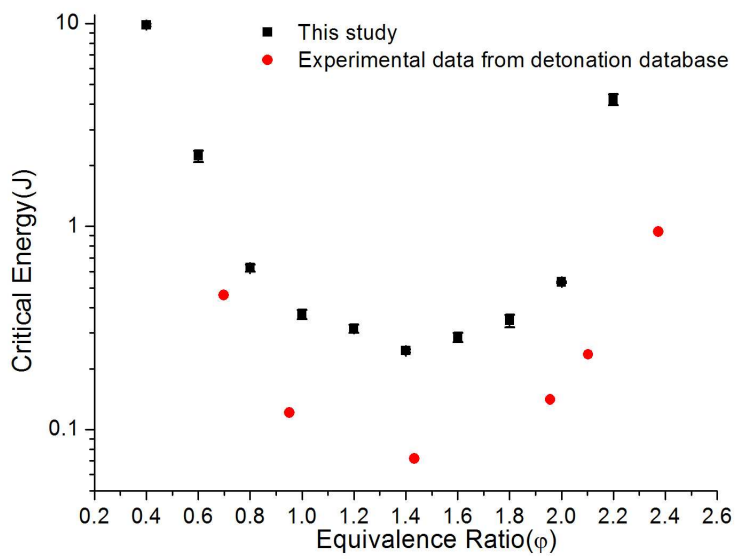

(b)

Fig. 2 Critical energy for $\mathrm{C}_{2} \mathrm{H}_{4}-3 \mathrm{O}_{2}$ mixtures as a function of a) initial pressure; and b) equivalence ratio at $p_{o}=100$ $\mathrm{kPa}$.

cell size $\lambda$ of all the undiluted mixtures considered in this study. The linear regression gives:

$R_{o}=26 \lambda$

with the coefficient of determination $R^{2}$ and the maximum deviation between the correlated values and the experimental data equal to 0.844 and $39.05 \%$, respectively. All the detonation cell size values for undiluted mixtures are interpolated/extrapolated using data from the Caltech Detonation Database [17]. It is worth noting that for undiluted mixture, the cell size is usually irregular particularly at low initial pressure, making the determination of a characteristic cell size difficult. The uncertainty in the cell size data for unstable mixtures can be as much as a factor of 2 . Taking these experimental errors into account, the goodness of the above linear regression appears reasonable.

Figure 6 shows the similar plot of explosion length $R_{o}$ as a function of detonation cell size $\lambda$ for the $\mathrm{C}_{2} \mathrm{H}_{2}$ $2.5 \mathrm{O}_{2}$ mixtures diluted with $50 \%, 65 \%$ and $70 \%$ argon. For argon diluted mixtures, the cell sizes are determined using correlations given in [18]. It appears that the relationship between the explosion length and the detonation

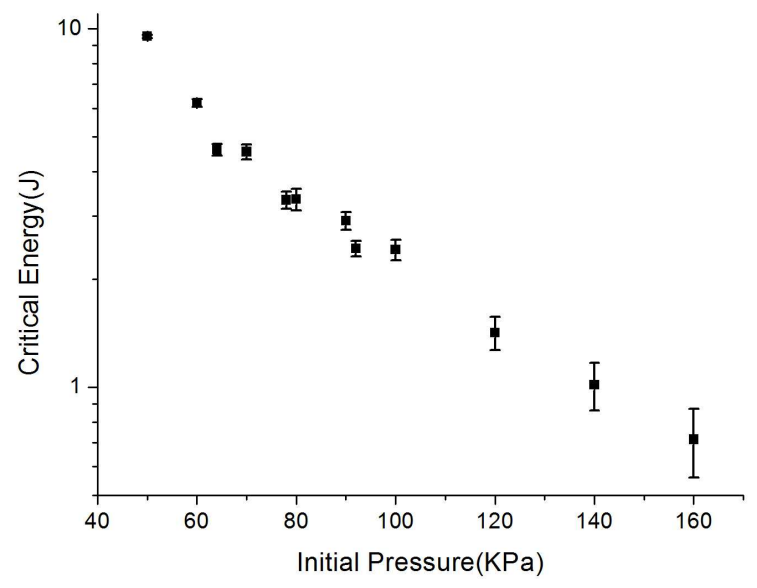

Fig. 3 Critical energy as a function of initial pressure for $\mathrm{C}_{3} \mathrm{H}_{8}-5 \mathrm{O}_{2}$ mixtures.

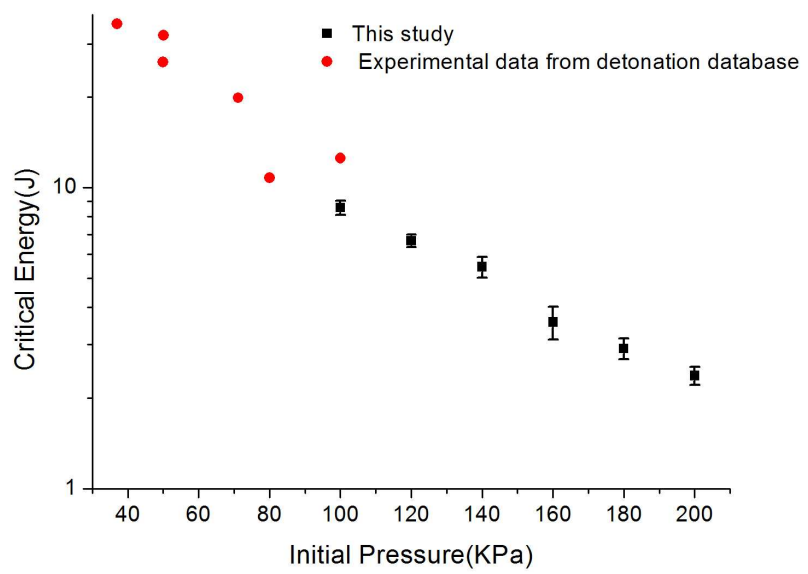

Fig. 4 Critical energy as a function of initial pressure for $2 \mathrm{H}_{2}-\mathrm{O}_{2}$ mixtures.

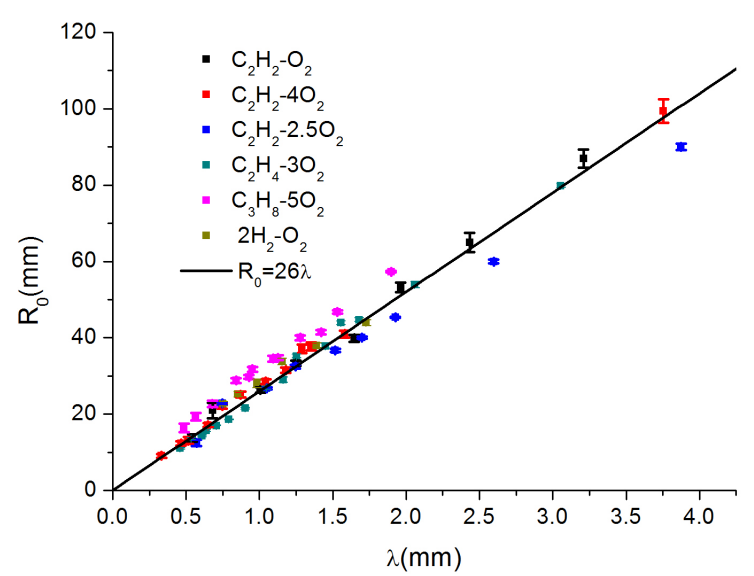

Fig. 5 Explosion length $R_{o}$ as a function of cell size $\lambda$ for undiluted mixtures. 


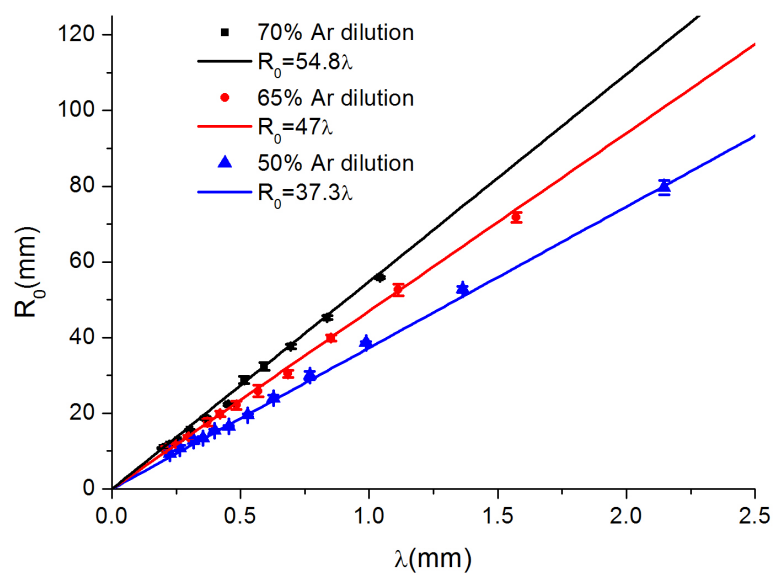

Fig. 6 Explosion length $R_{o}$ as a function of cell size $\lambda$ for $\mathrm{C}_{2} \mathrm{H}_{2}-2.5 \mathrm{O}_{2}$ mixtures diluted with $50 \%, 65 \%$ and $70 \%$ argon.

cell size still follows the linear scaling law. However, with an increasing amount of argon dilution, the proportionality factor also increases, i.e., to $37.3,47$ and 54.8 for $50 \%, 65 \%$ and $70 \%$ argon dilution, respectively. The results thus indicate that the characteristic length required for the reactive blast wave to evolve into a self-sustained detonation is relatively longer than that for the unstable detonations.

It is of interest to point out that the observation from the above scaling analysis agrees with that of the critical tube diameter problem [19]. For unstable detonations, the critical tube diameter follows well the universal correlation $d_{c}=13 \lambda$. While for highly stable detonations, the critical tube diameter can vary significantly from 20 $30 \lambda$. This led Lee to suggest that the distinct difference in the critical diameter phenomenon for the undiluted and highly diluted mixtures is related to the stability nature of the detonation front and failure mechanism [19]. For unstable detonations, failure is based on instabilities at which explosion centers are unable to form in the failure wave when it has penetrated to the charge axis. While for stable detonations, failure is caused by excessive curvature of the entire detonation front when the corner expansion waves have distributed the curvature over the detonation surface [19]. There appears a similarity between the present result and that of the critical tube diameter problem, which both suggest that the mechanism for both detonation propagation and initiation can be different in undiluted (unstable) and diluted (stable) combustible mixtures.

Knowing that for highly argon diluted mixtures, the detonation is relatively stable in that the reaction zone is at least piecewise laminar described by the ZND model and cellular instabilities play minor roles on the dynamics of the detonation [14-16], the explosion length may perhaps correlate better with the ZND induction zone length. Here Fig. 7 shows the explosion length $R_{o}$ as a function of ZND induction zone length for the $50 \%$, $65 \%$ and $70 \%$ argon diluted cases. All the ZND chemi-

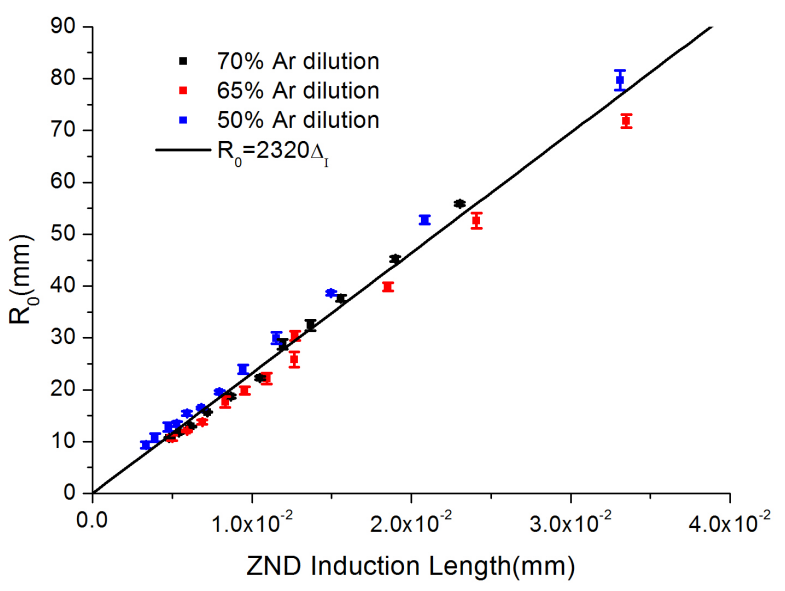

Fig. 7 Explosion length $R_{o}$ as a function of the ZND induction zone length for $\mathrm{C}_{2} \mathrm{H}_{2}-2.5 \mathrm{O}_{2}$ mixtures diluted with $50 \%$, $65 \%$ and $70 \%$ argon.

cal length scales are computed using the Chemkin package [20] with the Konnov chemical kinetic mechanism [21]. The Konnov mechanism had been validated and proven to be suitable for detonation simulation [22]. Results once again show a good linear relationship between these parameters for argon diluted mixtures given by:

$R_{o}=2320 \cdot \Delta_{I}$

For the above regression fit, the $R^{2}$ and the maximum deviation between the correlated values and the experimental data are respectively 0.998 and $20.48 \%$.

In contrast, an unique correlation or scaling between $R_{o}$ and $\Delta_{I}$ does not exist for undiluted mixtures as shown in Fig. 8. It is in fact expected since for unstable detonations, cellular instability plays a prominent role at the front and the dynamic structure departs from the ZND description.

\section{Concluding remarks}

In this paper, a new dataset of critical energy for direct initiation of detonations in some common gaseous fuel-oxygen mixtures is reported. These results should found applications in safety hazard assessment of these fuels and in developing and validating models for direct initiation of gaseous detonations. An analysis is also performed to look at the scaling between the explosion length $R_{o}$ that characterizes the blast wave and the chemical length scale that characterizes the detonation structure. Correlation analysis between the explosion length and the cell size confirms a linear dependence between these two parameters. It is found that $R_{o} \approx 26 \lambda$ is valid for all the undiluted mixtures considered in this study and the proportionality factors changes from 37.3, 47 and 54.8 respectively for $50 \%, 65 \%$ and $70 \%$ argon diluted $\mathrm{C}_{2} \mathrm{H}_{2}-2.5 \mathrm{O}_{2}$ mixtures. For 'stable' mixtures with high argon dilution, it is found the explosion length can 

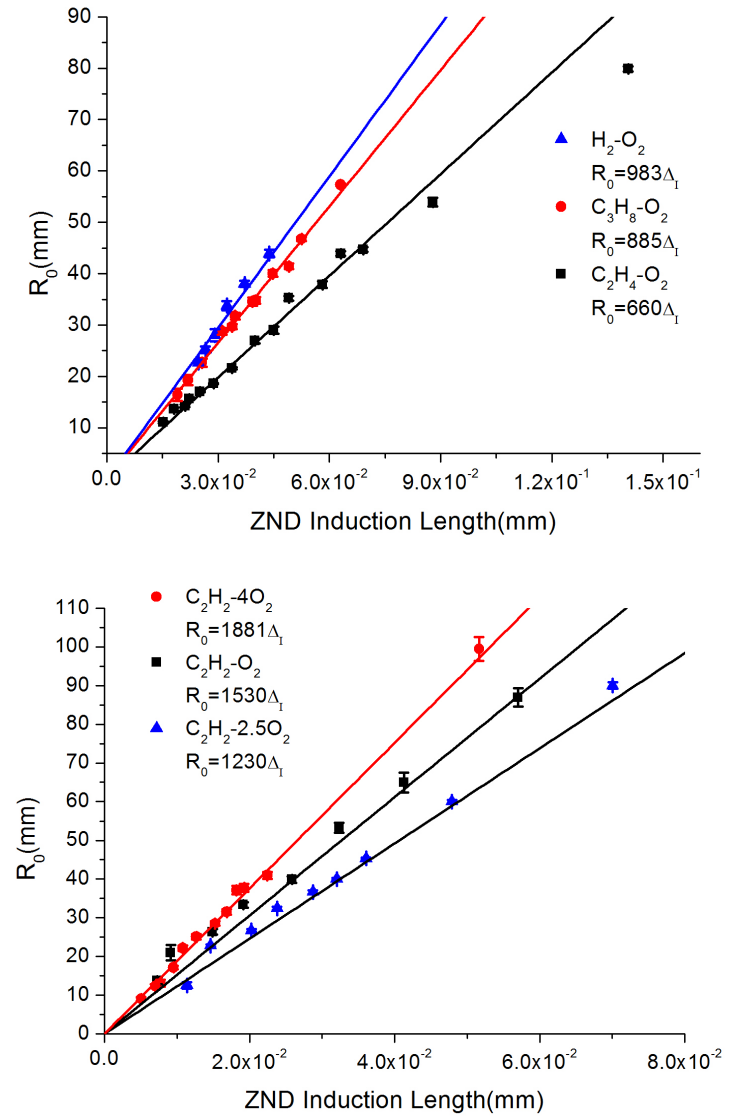

Fig. 8 Explosion length $R_{o}$ as a function of the ZND induction zone length for different undiluted combustible mixtures.

instead scale adequately with the ZND induction zone length. Results indicate that the explosion length $R_{O}$ is approximately $2320 \Delta_{I}$ for all the argon diluted mixtures considered in this work.

\section{References}

1. Matsui, H. and Lee, J.H.S.: On the measure of the relative detonation hazards of gaseous fuel-oxygen and air mixtures. Proc. Combust. Inst. 17, 1269-1280 (1978).

2. Lee, J.H.S.: Dynamic Parameters of Gaseous Detonations. Ann. Rev. Fluid Mech. 16, 311-336 (1984).

3. Lee, J.H.S., Higgins, A.J.: Comments on Criteria for Direct Initiation of Detonation. Phil. Trans. R. Soc. Lond. A 357, 3503-3521 (1999).

4. Vasil'ev, A.A., Mitrofanov, V.V. and Topchiyan, M.E.: Detonation waves in gases. Combust. Expl. Shock Waves 23(5), 605-623 (1987).

5. Zel'dovich, Y.B., Kogarko, S.M., Simonov, N.N.: An experimental investigation of spherical detonation in gases. Sov. Phys. Tech. Phys. 1, 1689-1713 (1957).

6. Lee, J.H.: Initiation of Gaseous Detonation. Ann. Rev. Phys. Chem 28, 75-104 (1977).

7. Murray, S.B. and Lee, J.H.S.: The influence of yielding confinement on large-scale ethylene-air detonations. Prog. Astro. Aero. 94, 80-103 (1984).

8. Radulescu, M.I., Higgins, A.J., Lee, J.H.S. and Murray, S.B.: On the explosion length invariance in direct initiation of detonation. Proc. Combust. Inst. 28, 637-644 (2000).
9. Knystautas, R., Lee, J.H.S.: On the effective energy for direct initiation of detonations. Combust. Flame 27, 221228 (1976).

10. Kamenskihs, V., Ng, H.D., Lee, J.H.S.: Measurement of critical energy for direct initiation of spherical detonations in high-pressure H2-O2 mixtures. Combust. Flame, 157(9), 1795-1799 (2010).

11. Zhang, B., Kamenskihs, V., Ng, H.D., Lee, J.H.S.: Direct blast initiation of spherical gaseous detonation in highly argon diluted mixtures. Proc. Combust. Inst. 33 (2), 22652271 (2010).

12. Zhang, B., Ng, H.D., Mével, R. and Lee, J.H.S.: Critical energy for direct initiation of spherical detonations in $\mathrm{H} 2 / \mathrm{N} 2 \mathrm{O} / \mathrm{Ar}$ mixtures. Int. J. Hydrogen Energy 36, 57075716 (2011).

13. Zhang, B., Ng, H.D. and Lee, J.H.S.: Measurement of effective blast energy for direct initiation of spherical gaseous detonations from high-voltage spark discharge. Revised manuscript submitted to Shock Waves SHOC381R1 (2011).

14. Radulescu, M.I. and Lee, J.H.S.: The failure mechanism of gaseous detonations: Experiments in porous wall tubes. Combust. Flame 131 (1-2), 29-46 (2002).

15. Radulescu, M.I., Ng, H.D., Varatharajan, B. and Lee, J.H.S.: The effect of argon dilution on the stability of acetylene-oxygen detonations. Proc. Combust. Inst. 29, 2825-2831 (2002).

16. Ng, H.D., Radulescu, M.I., Higgins, A.J., Nikiforakis, N. and Lee, J.H.S.: Numerical investigation of the instability for one-dimensional Chapman-Jouguet detonations with chain-branching kinetics. Combust.Theory Model. 9, 385401 (2005).

17. Kaneshige, M. and Shepherd, J.E.: Detonation database, GALCIT Technical Report FM97-8, http://www.galcit.caltech.edu/detn_db/html/, 1997.

18. Radulescu, M.I.: The Propagation and Failure Mechanism of Gaseous Detonations: Experiments in PorousWalled Tubes. Ph.D. thesis, McGill University, Canada (2003).

19. Lee, J.H.S.: On the critical tube diameter. In: J. Bowen, Editor, Dynamics of Exothermicity, Gordon and Breach, Amsterdam, Netherlands, pp. 321 (1996).

20. Kee, R.J., Rupley, F.M. and Miller, J.A.: A Fortran chemical kinetics package for the analysis of gas-phase chemical kinetics. Sandia National Laboratories report SAND89-8009, 1989.

21. Konnov, A.A.: Detailed reaction mechanism for small hydrocarbons combustion. Release 0.4, 1998, http://homepages.vub.ac.be/?akonnov/

22. Schultz, E. and Shepherd, J.E.: Validation of detailed reaction mechanisms for detonation simulation. GALCIT Technical Report FM99-5 (2000). 